\title{
Herausforderungen und Erwartungen an 3D-Technologien
}

\author{
Daniel Schubert ${ }^{1}$, Stephan Hörold ${ }^{2}$, Angelika C. Bullinger ${ }^{1}$, Heidi Krömker ${ }^{2}$
}

Professur Arbeitswissenschaft und Innovationsmanagement, TU Chemnitz ${ }^{1}$

Fachgebiet Medienproduktion, TU Ilmenau ${ }^{2}$

\section{Zusammenfassung}

3D-Technologien verbreiten sich in vielen Industriebranchen, wobei bisher die Herausforderungen und Erwartungen aus Sicht der Nutzer weitgehend unbekannt sind. Zur Identifizierung dieser Herausforderungen und Erwartungen wurden vier branchenspezifische Fokusgruppen mit Experten und eine Nutzerbefragung durchgeführt. Die Ergebnisse zeigen, dass eine generelle Akzeptanz gegenüber 3DTechnologien in den Branchen besteht. In Bezug zu den Herausforderungen und Erwartungen konnten jedoch keine signifikanten Unterschiede identifiziert werden. Daher kann auf Basis der Ergebnisse geschlossen werden, dass die Herausforderungen und Erwartungen bei der Entwicklung von 3DTechnologien in den untersuchten Branchen generell gleich sind, es allerdings branchenspezifische Abweichungen, wie bspw. die Berücksichtigung des Joy of Use oder des sozialen Ansehens, gibt.

\section{Einleitung}

3D-Technologien ${ }^{1}$ etablieren sich nach und nach in verschiedenen Branchen (Astor et al. 2013). Die Vorteile von 3D, wie bspw. eine bessere Diagnostik durch Tiefeninformationen in 3D-Darstellungen (z. B. in der Medizin oder bei der Wartung von Maschinen) oder auch die Bedienung von Geräten durch Gesten, verdeutlichen den Nutzen der 3D-Technologien (Bowman et al. 2005). Damit dieser Nutzen auch von den Nutzern verstanden werden kann, ist es besonders wichtig, Erwartungen und Herausforderungen aus Sicht zukünftiger Nutzer, insbesondere im Kontext der Mensch-Maschine-Interaktion, zu analysieren.

Im Rahmen der Forschung werden die Branchen Produktion, Sicherheit, Gesundheit und Mobilität untersucht, um herauszufinden, welche Gemeinsamkeiten und Unterschiede in den

1 3D-Technologien umfassen in diesem Beitrag Technologien, die es ermöglichen räumliche Daten von Personen und Objekten systemgestützt zu erfassen und zu verarbeiten und den Nutzern die Interaktion mit 3D-Objekten ermöglichen. 
Branchen von den Nutzern erwartet werden. Ziel ist es, auf dieser Basis den Faktor Mensch in den Fokus der Entwicklung zu stellen.

Um die Forschungsfrage zu beantworten, wird der Beitrag in einen Überblick über die Methodik, die Präsentation der Ergebnisse und eine Zusammenfassung mit Ausblick gegliedert.

\section{Methode}

Zur Eruierung der Erwartungen, Herausforderungen und Unterschiede wurden vier branchenspezifische Fokusgruppen mit standardisiertem Vorgehen und im Anschluss auf Basis dieser Erkenntnisse eine Onlinebefragung durchgeführt. Die Durchführung erfolgte an vier Standorten mit 27 Experten aus wissenschaftlichen Einrichtungen und Unternehmen der genannten Branchen. Die Leitthemen der Fokusgruppen umfassen die Einflüsse auf den derzeitigen Entwicklungsprozess und die erwarteten Herausforderungen für die zukünftigen Nutzer der 3D-Technologie. Neben den Fokusgruppen wurde eine Onlinebefragung $(\mathrm{N}=50)$ mit zukünftigen Nutzern von 3D-Technologien durchgeführt. Um das Thema 3DTechnologien verständlich aufzubereiten, wurde die Komplexität dieser Technologien auf die Bereiche Erfassung, Verarbeitung, Eingabe und Ausgabe heruntergebrochen, da diese vier Bereiche die Einflussparameter in einem Mensch-Maschine-System darstellen (Bubb 1993) und somit die Grundlage für eine nutzerzentrierte Gestaltung bilden.

\section{Branchenvergleich}

Die Analysen zeigen, dass die vier Branchen durch ähnliche Erwartungen und Herausforderungen charakterisiert werden. Die Grundlage für die Auswertung bilden die Ergebnisse der Fokusgruppen sowie der Nutzerbefragung. Im Rahmen der Auswertung wird nach Erfassung und Verarbeitung durch technische Systeme sowie nach Eingabe und Ausgabe durch die Nutzer unterschieden.

\subsection{Erfassung und Verarbeitung}

Die Ergebnisse der Nutzerbefragung zeigen, dass in allen vier Branchen eine grundsätzliche Verbesserung des Detailgrades, der Verlässlichkeit und der Geschwindigkeit sowie allgemein die Erfassung und Verarbeitung von mehr Daten erwartet werden. Wie Abbildung 1 zeigt, wird lediglich für die Branche Sicherheit ein negativer Einfluss auf die Geschwindigkeit erwartet. Die Ergebnisse der Fokusgruppen zeigen, dass die Herausforderungen des Einsatzes von 3D-Technologien in der Erfassung und Verarbeitung in den Branchen eine hohe Übereinstimmung aufweisen. Typische Herausforderungen in diesen Teilbereichen sind die steigende technische Komplexität, der erhöhte Lernaufwand zur Systembedienung und zur Qualitätsbeurteilung sowie die Interpretation von Daten durch Mensch und Maschine. 


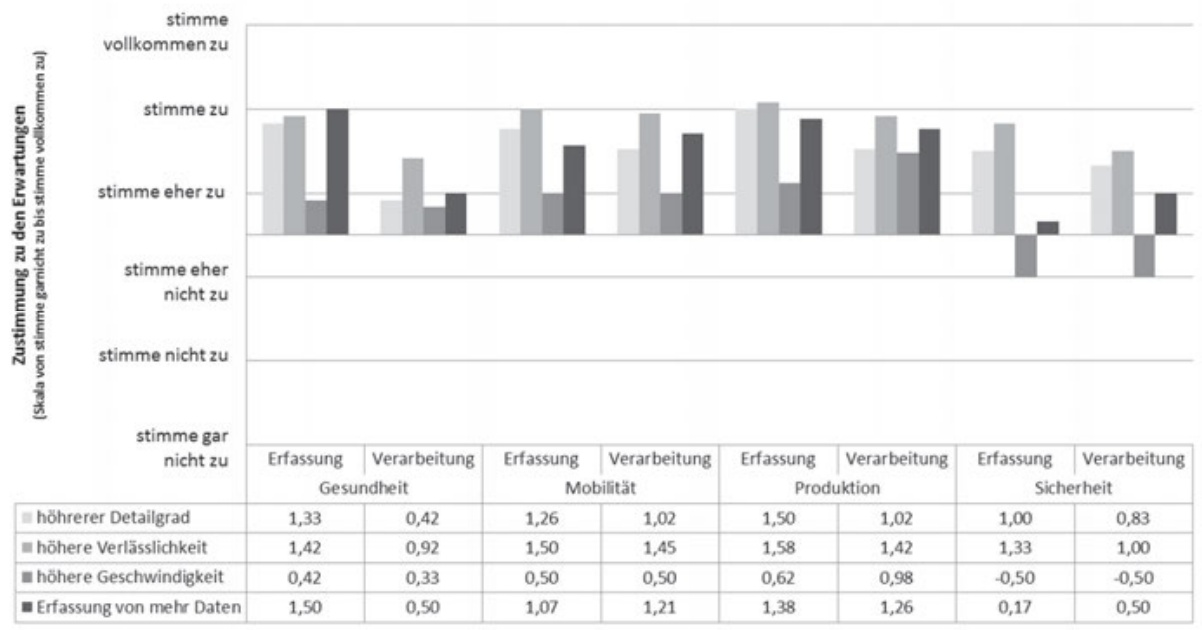

Abbildung 1: Erwartungen an die Erfassung und Verarbeitung mit 3D-Technologien (Mittelwerte)

\subsection{Eingabe und Ausgabe}

Aus Sicht der Nutzer wird in allen Branchen eine zuverlässige sowie entspannte Bedienung erwartet. Mit Ausnahme der Mobilität wird die Wichtigkeit eines höheren Ansehens sowie der Spaß an der Bedienung eher gering eingeschätzt, wie Abbildung 2 zeigt.

Zudem zeigt der Vergleich zwischen 2D und 3D-Technologien für die Ein- und Ausgabe, dass die 3D-Technologien tendenziell positiv bewertet werden. Lediglich für die Einfachheit der 3D-Eingabesysteme ist die Beurteilung negativ und sieht die 2D-Technologien positiver.

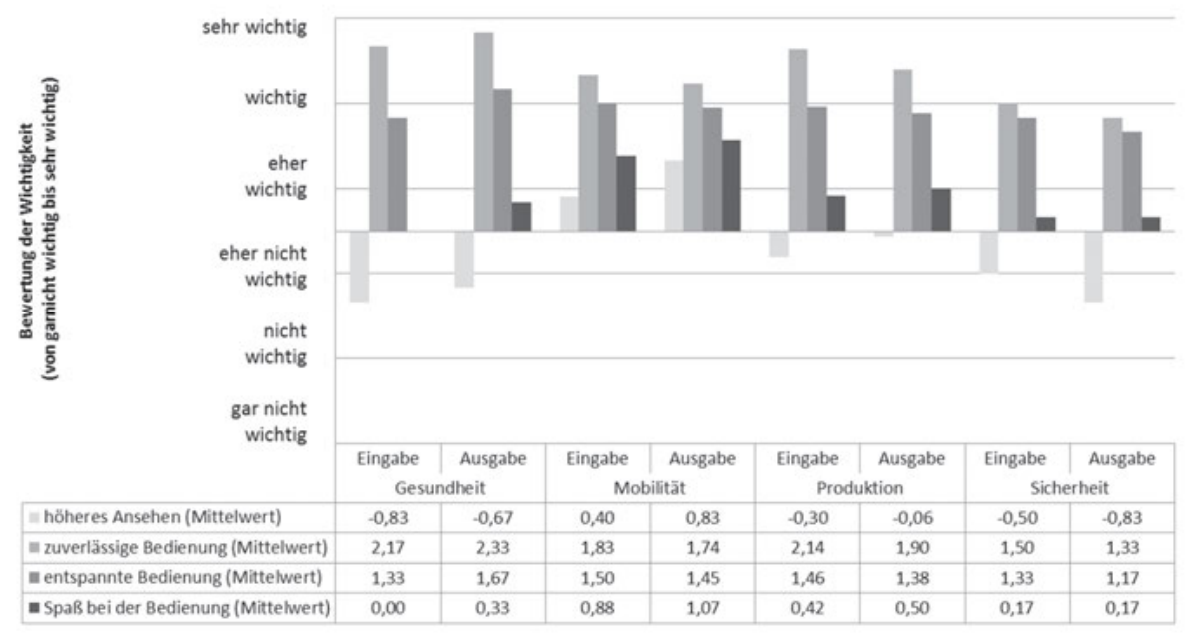

Abbildung 2: Erwartungen an die Ein- und Ausgabe mit 3D-Technologien (Mittelwerte) 
Den Erwartungen an die 3D-Technologien stehen die in den Fokusgruppen mit Experten identifizierten übergreifenden Herausforderungen gegenüber:

- Steigerung der Akzeptanz durch Vertrauen und Mehrwert der 3D-Technologie

- Nahtlose Integration der 3D-Technologien in Systeme und Prozesse

- Nutzerorientierte Entwicklung von 3D-Interaktionstechniken und -metaphern

- Standardisierung zur leichteren Einbindung und Nutzung

\section{Zusammenfassung und Ausblick}

Die Ergebnisse zeigen, dass eine grundlegende Akzeptanz und positive Erwartung gegenüber 3D-Technologien bei den Experten und Nutzern in den vier Branchen vorliegt. Allerdings konnten in allen Teilbereichen Herausforderungen der Mensch-Technik-Interaktion, z. B. 2D/3D-Mischinteraktion, Skalierungseffekte, geringe Erwartungskonformität und Lernförderlichkeit, identifiziert werden. Für die Weiterentwicklung der 3D-Technologien zeigen die Ergebnisse, dass auch neue Interaktionsmetaphern (Schubert et al. 2015) und systematische Lernprozesse entwickelt werden müssen. Zudem konnte in der Analyse festgestellt werden, dass sich unterschiedliche Rahmenbedingungen, z. B. Integrationsprozesse von 3DTechnologien in der Produktion, sowie unterschiedliche Nutzungskontexte und Entwicklungsprozesse auf diese Erwartungen und Herausforderungen auswirken. Dies muss in folgenden Arbeiten adressiert werden, um 3D-Technologien nutzerzentriert zu integrieren.

\section{Acknowledgement}

Dieser Beitrag wurde durch die Unterstützung des Bundesministeriums für Bildung und Forschung ermöglicht (Projekt: 3Dsensation - Basisprojekt Faktor Mensch, FKZ 03ZZ0403).

\section{Kontaktinformationen}

\begin{tabular}{|l|l|}
\hline Dipl.-Ing. Stephan Hörold & Dipl.-Wirtsch.-Ing Daniel Schubert \\
Technische Universität Ilmenau & Technische Universität Chemnitz \\
98693 Ilmenau & 09107 Chemnitz \\
E-Mail: stephan.hoerold@tu-ilmenau.de & E-Mail: daniel.schubert@ mb.tu-chemnitz.de \\
\hline
\end{tabular}

\section{Literaturverzeichnis}

Astor, M., Glöckner, U., Klose, G., Plume, A., Schneidenbach, T., von Lukas, U., Bechtold, I., Ruth, T., Jarowinsky \& M., Bartels, H. (2013). Marktperspektiven von 3D in industriellen Anwendungen. Berlin.

Bowman, D. A., Kruijff, E., LaViola, J. \& Poupyprev, I. (2005). 3D User Interfaces - Theory and Practice. Boston: Addison-Wesley.

Bubb, H. (1993). Systemergonomie. In Schmidtke, H. (Hrsg.): Ergonomie. München: Carl Hanser.

Schubert, D., Jentsch, M. \& Bullinger, A. C. (2015). Je eher, je besser: Nutzerzentriertes Mobility Engineering für 3D-Fahrer-Fahrzeug-Interaktion. In Bullinger, A. C. (Hrsg.): Mensch 2020 - transdisziplinäre Perspektiven. Chemnitz: Verlag aw\&I Wissenschaft und Praxis. 